\title{
Mitochondrial Genetic Disorders: Challenges \& Opportunities
}

\author{
Vaibav Saxena* \\ Boston Biomedical, Inc, Cambridge, MA, USA
}

Received: December 18, 2014, Accepted: January 30, 2015, Published: February 03, 2015

*Corresponding author: Vaibhav Saxena, Ph.D., Boston Biomedical, Inc, Cambridge, MA, Tel: 917-455-6857; Email: vaibhavsx@gmail.com

\begin{abstract}
Mitochondria play a key role in the normal functioning of the cell. The mutations in the mitochondrial genome produce faulty proteins thus hampering its normal activity. In the past few years, though several genetic disorders have been identified due to the defect in the mitochondrial DNA (mtDNA), there is no significant clinical progress yet to treat such disorders. The foremost reasons being the extreme complexities associated with such genetic defects; the presence of several copies of mtDNA in a mitochondrion, the co-existence of wild-type and mutated mtDNA, symptoms matching with other pathological conditions, motherly inheritance and somatic nature of these mtDNA mutations. Our incomplete understanding of the mitochondrial transcription machinery and mitochondrial genetic code variations add to the woes. To further our understanding in this field, it is extremely critical to develop mitochondria-specific delivery systems and mitochondria-targeted reporter gene systems.
\end{abstract}

Keywords: Mitochondria; Mitochondrial DNA (mtDNA); Mitochondrial Genetic Disorders; Nanocarrier Delivery Systems; Subcellular Targeting

\section{Introduction}

Mitochondria perform some of the most vital functions in the life cycle of a cell; it regulates thermogenesis, calcium homeostasis [1], it's the site for the ATP production and also plays a key role in apoptosis [2], which is a mechanism of programmed cell death. The proteins required for the successful functioning of the mammalian mitochondrial machinery are synthesized in two separate genomes. While, the majority of the mitochondrial proteins are synthesized in the nuclear genome, expressed in the cytoplasm and then gets transferred to the mitochondria by a complex protein import machinery [3,4]; few, yet extremely important proteins get synthesized and expressed exclusively in the mitochondrial matrix. So, it is quite imperative that any defect in the mitochondrial genome would result in the production of the faulty proteins inside the mitochondrial matrix and thus, would have a direct impact on the overall performance of the mitochondria.

\section{The Mitochondrial Genome}

The mitochondrial genome is a closed, circular double stranded DNA (dsDNA) molecule, with a size of about $16.5 \mathrm{~kb}$ [5]. It is devoid of introns and the only non-coding portion it has, is the D-Loop, a $1 \mathrm{~kb}$ region, which is essentially the site for the heavy and light strand promoters and the transcription initiation sites. Overall, the mitochondrial genome encodes for 2 ribosomal RNA (rRNAs) (12S and 16S), 22 Transfer RNAs (tRNAs) and 13 protein sub-units of OXPHOS complexes (I, III, IV \& V) [5].

\section{Mutations in the Mitochondrial Genome}

Due to the close proximity of the mitochondrial genome to the respiratory chain complex producing ATP, it is believed to be more susceptible to get damaged by the reactive oxygen species (ROS) than nuclear DNA. In addition to this, the poorly developed mitochondrial DNA (mtDNA) repair machinery and the lack of protective histones, unlike in nuclear genome are also believed to be the major factors for high mutation rate [6-8].

The mutations found in mitochondria are either point mutations (single base substitutions) or simply large-scale deletions of nucleotides [9]. The diseases found to be associated with such mutations in mtDNA includes neurodegenerative and neuromuscular diseases like Mitochondrial Encephalomyopathy Lactic Acidosis and Stroke-like episodes (MELAS), Cardiac Myopathy (CM), Mitochondrial Encephalomyopathy, Lactic Acidosis and Stroke-like episodes (MELAS), Leber's hereditary Optic Neuropathy (LHON), Neuropathy, Ataxia and Retinitis Pigmentosa (NARP) syndrome, Chronic Progressive External Ophthalmoplegia (CPEO) and Leigh's disease to name few. Recently, the mitochondrial genetic mutations have also been linked to the cancer and aging process as well [10-11].

\section{Complexities of the Mitochondrial Genetic Disorders}

The clinical diagnosis of the mitochondrial genetic disorders has been difficult, the major reason being the matching of its symptoms with some commonly occurring diseases and hence difficult to detect [12]. This further gets complicated by the fact that, a mitochondrion posses several copies of mtDNA polyploidy [13]. Few of these mtDNA undergo mutation and become pathogenic, which then subsequently co-exist with a wild-type healthy mtDNA within the same cell or tissue of an individual, a condition termed as heteroplasmy [14]. It is when the critical threshold levels of the heteroplasmy get exceeded, the 
expression of biochemical and clinical defects are observed. The maternal inheritance and stochastic segregation of mtDNA along with an incomplete knowledge of the mitochondrial genome transcription machinery further add to the complexity of mtDNA diseases $[15,16]$.

\section{Biological barriers}

One important facet of targeted-delivery is the specific delivery of cargo to its intended location, followed by its action. In this case, we take one step forward and discuss the idea to develop a safe non-viral, nanocarrier system, which not only delivers the bio-molecules to the target cells but to the specific organelle of the cell, in this case, a mitochondrion. However, to achieve the same, there are several cellular \& sub-cellular barriers that a delivery system has to overcome [17]. The extracellular barriers include the stability of the nanocarrier in blood serum, ability to escape the reticuloendothelial system (RES), and binding to target cell types. Intracellularly, the nanocarrier must be able to escape the endo-lysosomal barrier, traffic its way inside the highly viscous cytosolic matrix, and binds to the target organelle mitochondrion. The final step in this process is overcoming the mitochondrial membrane barrier, and the release of bioactive molecules inside the mitochondrial matrix. In case of the mtDNA specific genes, they further needs to be taken up the mitochondrial genome for the successful production of a functional protein.

\section{DNA delivery to mitochondria}

To deliver DNA specifically to mitochondria, several nanocarrier-mediated targeting approaches have been developed \& tested on cultured cells. The first approach is the use of delivery vehicles having mitochondrial affinity, such as DQAsomes, which are liposome-like vesicles [18]. They have been successful in delivering DNA and short DNA-conjugated sequences to the mitochondria based on its DNA condensation and mitochondriotropic properties [19,20]. Other technique was the utilization of mitochondriotropic cationic amphiphiles, like triphenyl phosphonium ion (TPP), which utilizes the high membrane potential of the mitochondrial membrane and has been shown to successfully deliver PNA oligomer to the mitochondria after conjugation to TPP [21]. In a less toxic alternative, mitochondriotropic liposome's modified by amphiphilic cations displayed cargo delivery to mitochondria and a promise for gene delivery applications [22]. The liposome's prepared with isolated mitochondrial fraction were also shown to deliver DNA to the mitochondria of mammalian cells [23]. Based on the ability of certain lipids to fuse with the mitochondrial membranes, another class of nanocarrier, MITO-Porters and more recently dual function MITO-Porters, having both endosome and mitochondria fusogenic envelope showed the delivery of GFP to human cells $[24,25]$. In all the above stated cases, though the system ensures intracellular delivery and subsequently promoted enhanced colocalization of the delivered DNA with mitochondria; whether the DNA actually got inside the organelle needs to be established.

In addition to this, the mitochondrial protein import pathway has also been exploited to direct DNA into the mitochondria [26]. In one of the notable studies, the complex of mitochondrial targeting peptide conjugated to peptide nucleic acid (PNA), and then annealed to DNA oligonucleotide were shown to be capable of uptake by isolated mammalian mitochondria and the mitochondria of cultured mammalian cells, when permeabilized to allow cytosolic uptake [27]. In protofection-based strategy, a complex of DNA loaded with mitochondrial transcription factor-A (TFAM), combined with mitochondrial targeting sequence (MTS) and protein transduction domain (TD) was successful in partially restoring mitochondrial functions for Parkinson's disease cybrid model cells [28].

More recently, a new set of nanoparticle delivery systems, engineered to specifically target mitochondria have been reported in recent studies. This includes biodegradable polymeric nanoparticle platforms, such as PLGA-PEG nanoparticles, surface modified with TPP moiety [29,30]; mitochondrial targeting gold peptides [31]; TPP conjugated poly (amidoamine) dendrimers [32]; multifunctional enveloped mesoporous silica nanoparticles (MSNs) modified with TPP, peptides and coated with $\mathrm{pH}$-sensitive PEG-PLL (DMA) polymer [33]; rhodamine-based plasmid DNA nanoparticles [34] and functional DOX-nanoparticles prepared with folate-terminated polyrotaxanes (FPRs) and dequalinium (DQA) [35]. These nanocarrier formulations represent some novel techniques delivering various small molecules, peptides \& DNA to mitochondria and along with previously mentioned delivery systems offer promise and potential to routinely target mitochondria as a sub-cellular target.

\section{Conclusion}

Based on all the above-discussed factors, it is imperative that to further our understanding regarding the mitochondrial genome and for its plausible therapeutic application to treat its genetic disorders in the future, first and foremost, there is a need to develop robust mitochondria-targeted nanocarrier devices, which could deliver the therapeutic genes and drugs specifically \& routinely to the mitochondria. Second, there is a need to develop sophisticated bioassays, which could actually establish the delivery of DNA inside the mitochondria. Lastly, there is also an urgent need to develop a mitochondria-specific functional reporter system for easy expression and identification inside mitochondria.

\section{References}

1. McFarland R, Taylor RW, Turnbull DM. Mitochondrial disease-its impact, etiology, and pathology. Curr Top Dev Biol. 2007; 77: 113-155.

2. Grad JM, Cepero E, Boise LH. Mitochondria as targets for established and novel anti-cancer agents. Drug Resist Updat. 2001; 4(2): 85-91.

3. Schatz G, Dobberstein B. Common principles of protein translocation across membranes. Science. 1996; 271(5255): 1519-1526.

4. Hoogenraad NJ, Ward LA, Ryan MT. Import and assembly of proteins into mitochondria of mammalian cells. Biochim Biophys Acta. 2002; 1592(1): 97-105.

5. Taanman JW. The mitochondrial genome: structure, transcription, translation and replication. Biochim Biophys Acta. 1999; 1410(2): 103-123.

6. Scheffler IE. A century of mitochondrial research: achievements and perspectives. Mitochondrion. 2001; 1(1): 3-31. 
7. Thyagarajan B, Padua RA, Campbell C. Mammalian mitochondria possess homologous DNA recombination activity. J Biol Chem. 1996; 271(44): 27536-27543.

8. Mason PA, Matheson EC, Hall AG, Lightowlers RN. Mismatch repair activity in mammalian mitochondria. Nucleic Acids Res. 2003; 31(3): 1052-1058.

9. Enns GM. The contribution of mitochondria to common disorders. Mol Genet Metab. 2003; 80(1-2): 11-26.

10. Shokolenko IN, Wilson GL, Alexeyev MF. Aging: A mitochondrial DNA perspective, critical analysis and an update. World J Exp Med. 2014; $4(4): 46-57$.

11. D’Souza GG, Wagle M.A, Saxena V, Shah A. Approaches for targeting mitochondria in cancer therapy. Biochim Biophys Acta. 2011; 1807(6): 689-96. doi: 10.1016/j.bbabio.2010.08.008.

12. Munnichand A, Rustin P. Clinical spectrum and diagnosis of mitochondrial disorders. Am J Med Genet. 2001; 106(1): 4-17.

13. Satoh M, Kuroiwa T. Organization of multiple nucleoids and DNA molecules in mitochondria of a human cell. Exp Cell Res. 1991; 196(1) 137-140.

14. Solignac M, Monnerot M, Mounolou JC. Mitochondrial DNA heteroplasmy in Drosophila mauritiana. Proc Natl Acad Sci USA. 1983; 80(22): 6942-6946.

15. Pulkesand T, Hanna MG. Human mitochondrial DNA diseases. Adv Drug Deliv Rev. 2001; 49(1-2): 27-43.

16. Naviaux RK. Developing a systematic approach to the diagnosis and classification of mitochondrial disease. Mitochondrion. 2004; 4(5-6): 351-361.

17. Cho K, Wang X, Nie S, Chen ZG, Shin DM. Therapeutic nanoparticles for drug delivery in cancer. Clin Cancer Res. 2008; 14 (5): 1310-1316. doi: 10.1158/1078-0432.CCR-07-1441.

18. Weissig V, Lasch J, Erdos G, Meyer HW, Rowe TC, Hughes J. DQAsomes: a novel potential drug and gene delivery system made from Dequalinium. Pharm. Res. 1998; 15(2): 334-337.

19. D'Souza GG, Rammohan R, Cheng SM, Torchilin VP, Weissig V DQAsome-mediated delivery of plasmid DNA toward mitochondria in living cells. J Control Release. 2003; 92(1-2): 189-197.

20. D’Souza GG, Boddapati SV, Weissig V. Mitochondrial leader sequenceplasmid DNA conjugates delivered into mammalian cells by DQAsomes co-localize with mitochondria. Mitochondrion. 2005; 5(5): 352-358.

21. Muratovska A, Lightowlers RN, Taylor RW, Turnbull DM, Murphy MP, Wilce JA, et al. Targeting peptide nucleic acid (PNA) oligomers to mitochondria within cells by conjugation to lipophilic cations: implications for mitochondrial DNA replication, expression and disease. Nucleic Acids Res. 2001; 29(9): 1852-1863.

22. Boddapati SV, Tongcharoensirikul P, Hanson RN, D’Souza GG Torchilin VP, Weissig V. Mitochondriotropic liposomes. J Liposome Res. 2005; 15(1-2): 49-58.
23. Wagle MA, Martinville LE, D’Souza GG. The utility of an isolated mitochondrial fraction in the preparation of liposomes for the specific delivery of bioactives to mitochondria in live mammalian cells. Pharm Res. 2011; 28(11): 2790-2796. doi: 10.1007/s11095-011-0531-5.

24. Yamada Y, Akita H, Kamiya H, Kogure K, Yamamoto T, Shinohara Y, et al. MITO-Porter: a liposome-based carrier system for delivery of macromolecules into mitochondria via membrane fusion. Biochim Biophys Acta. 2008; 1778(2): 423-432.

25. Yamada Y, Harashima H. Delivery of bioactive molecules to the mitochondrial genome using a membrane-fusing, liposome-based carrier, DF-MITO-Porter. Biomaterials. 2012; 33(5): 1589-1595. doi: 10.1016/j.biomaterials.2011.10.082.

26. Becker T, Bottinger L, Pfanner N. Mitochondrial protein import: from transport pathways to an integrated network. Trends Biochem Sci. 2012; 37(3): 85-91. doi: 10.1016/j.tibs.2011.11.004.

27. Flierl A, Jackson C, Cottrell B, Murdock D, Seibel P, Wallace DC. Targeted delivery of DNA to the mitochondrial compartment via import sequence-conjugated peptide nucleic acid. Mol Ther. 2003; 7(4): 550-557.

28. Keeney PM, Quigley CK, Dunham LD, Papageorge CM, Iyer S, Thomas $\mathrm{RR}$, et al. Mitochondrial gene therapy augments mitochondrial physiology in a Parkinson's disease cell model. Hum Gene Ther. 2009; 20(8): 897-907. doi: 10.1089/hum.2009.023.

29. Marrache S, Dhar S. Engineering of blended nanoparticle platform for delivery of mitochondria-acting therapeutics. Proc Natl Acad Sci. 2012; 109 (40): 16288-93. doi: 10.1073/pnas.1210096109.

30. Marrache S, Pathak RK, Dhar S. Detouring of cisplatin to access mitochondrial genome for overcoming resistance. Proc Natl Acad Sci U S A. 2014; 111 (29): 10444-9. doi: 10.1073/pnas.1405244111.

31. Ma X, Wang X, Zhou M, Fei H. A mitochondria-targeting gold-peptide nanoassembly for enhanced cancer-cell killing. Adv Healthc Mater. 2013; 2 (12): 1638-43. doi: 10.1002/adhm.201300037.

32. Biswas S, Dodwadkar NS, Piroyan A, Torchilin VP. Surface conjugation of triphenylphosphonium to target poly(amidoamine) dendrimers to mitochondria. Biomaterials. 2012; 33 (18): 4773-82. doi: 10.1016/j. biomaterials.2012.03.032.

33. Luo GF, Chen WH, Liu Y, Lei Q, Zhuo RX, Zhang XZ. Multifunctional enveloped mesoporous silica nanoparticles for suncellular co-delivery of drug and therapeutic peptide. Sci Rep. 2014; 4: 6064. doi: 10.1038/ srep06064.

34. Santos J, Sousa F, Queiroz J, Costa D. Rhodamine based plasmid DNA nanoparticles for mitochondrial gene therapy. Colloids Surf B Biointerfaces. 2014; 121: 129-140. doi: 10.1016/j. colsurfb.2014.06.003.

35. Wang H, Yin H, Yan F, Sun M, Du L, Peng W, et al. Folate-mediated mitochondrial targeting with doxorubicin-polyrotaxane nanoparticles overcomes multidrug resistance. Oncotarget. 2014. 